\title{
Genome-level comparisons provide insight into the phylogeny and metabolic diversity of species within the genus Lactococcus
}

\author{
Jie Yu(D, Yuqin Song, Yan Ren, Yanting Qing, Wenjun Liu and Zhihong Sun ${ }^{*}$
}

\begin{abstract}
Background: The genomic diversity of different species within the genus Lactococcus and the relationships between genomic differentiation and environmental factors remain unclear. In this study, type isolates of ten Lactococcus species/subspecies were sequenced to assess their genomic characteristics, metabolic diversity, and phylogenetic relationships.

Results: The total genome sizes varied between 1.99 (Lactococcus plantarum) and 2.46 megabases (Mb; L. lactis subsp. lactis), and the G + C content ranged from 34.81 (L. lactis subsp. hordniae) to 39.67\% (L. raffinolactis) with an average value of $37.02 \%$. Analysis of genome dynamics indicated that the genus Lactococcus has an open pan-genome, while the core genome size decreased with sequential addition at the genus and species group levels. A phylogenetic dendrogram based on the concatenated amino acid sequences of 643 core genes was largely consistent with the phylogenetic tree obtained by 165 ribosomal RNA (rRNA) genes, but it provided a more robust phylogenetic resolution than the 16S rRNA gene-based analysis.
\end{abstract}

Conclusions: Comparative genomics indicated that species in the genus Lactococcus had high degrees of diversity in genome size, gene content, and carbohydrate metabolism. This may be important for the specific adaptations that allow different Lactococcus species to survive in different environments. These results provide a quantitative basis for understanding the genomic and metabolic diversity within the genus Lactococcus, laying the foundation for future studies on taxonomy and functional genomics.

Keywords: Lactococcus, Comparative genomics, Carbohydrate metabolism, Phylogeny

\section{Background}

Lactococcus is a genus of catalase-negative, gram-positive, non-motile, facultative anaerobic bacteria that typically inhabit animals, plants, and their related products, particularly fermented products; they are generally considered nonpathogenic toward humans [1]. Lactococcus species have variable and abundant nutritional requirements. They usually grow within a temperature range of $10-40{ }^{\circ} \mathrm{C}$, although some species are capable of growing at temperatures as low as $7{ }^{\circ} \mathrm{C}$ over a prolonged incubation period of 10-14 days [2]. Most Lactococcus species can grow in 4.0\%

\footnotetext{
* Correspondence: sunzhihong78@163.com

Key Laboratory of Dairy Biotechnology and Engineering, Ministry of Education, Key Laboratory of Dairy Products Processing, Ministry of Agriculture, Inner Mongolia Agricultural University, Hohhot, China
}

$(w / v) \mathrm{NaCl}$; in media, they grow best at $\mathrm{pH} 7.0$ and cease to grow if the $\mathrm{pH}$ drops to 4.5 [3].

Lactococcus lactis was the first species in the genus to be identified (by Joseph Lister in 1873) and then it was later renamed Streptococcus lactis [1]. Schleifer et al. defined the genus Lactococcus as distinct from the genus Streptococcus [4]. To date, there are 12 recognized species with four further subspecies in the genus (http:// www.bacterio.net/lactococcus.html, December 2016). Some species, especially L. lactis, are important in the manufacturing of fermented milk products and some species produce antimicrobial compounds, such as bacteriocins, nisin, and lactococcin $[5,6]$, and recombinant proteins [7]. Some probiotic foods contain these species, and there have been more studies regarding the relationships between these probiotic-containing functional foods and the maintenance of human intestinal health 
[8, 9]. One species, Lactococcus garvieae, is a major pathogen of fish causing fatal hemorrhagic septicemia [10]. However, L. garvieae has also been reported as a common component of fermented dairy manufactured from raw milk [11-14], and phenotypically it is closely related to L. lactis.

The number of studies using high-throughput sequencing to study bacterial metabolism, evolution, and speciation within genera has increased markedly with the advances and decreasing costs of sequencing technologies [15-17]. Whole genomic sequences allow reconstruction of reliable phylogenies based on much larger sequence data sets than previously available. This makes it possible to gain an understanding of the genomic diversification of entire genera in great detail [18]. The first genome of a lactic acid bacterium (LAB), L. lactis isolate IL1403, was sequenced in 2001 [19]. Subsequently, there have been increasing reports on whole genome sequences of LAB. However, of the 16 currently recognized species/subspecies in the genus Lactococcus, only seven have been fully sequenced (National Center for Biotechnology information [NCBI]): L. lactis subsp. lactis, L. lactis subsp. cremoris, $L$. garvieae, $L$. raffinolactis, $L$. plantarum, $L$. fujiensis, and L. piscium [20-23]. With many new species being identified in the genus Lactococcus, a genomic view could provide important information to understand bacterial evolution and adaptation to different environments. Moreover, comparative genomic of species within the same genus can reveal the genomic diversity within a genus and provide insights into how environmental conditions affect the evolution of different species.

In the present study, the genomes of ten type species/ subspecies in the genus Lactococcus were sequenced. We then used comparative genomic approaches to define the pan-genome, core genome, and unique genes; assess genetic diversity and species taxonomy; infer phylogenetic relationships; and study the mechanisms of carbohydrate metabolism.

\section{Methods}

\section{Bacterial isolates, DNA extraction and carbohydrate} utilization

The ten type isolates of Lactococcus species/subspecies, as defined by the LPSN (http://www.bacterio.net/) were collected from the German Collection of Microorganisms and Cell Cultures (DSMZ; Table 1). These isolates were cultured in M17 broth (Oxoid Ltd., Basingstoke, UK) at $37^{\circ} \mathrm{C}$ for $24 \mathrm{~h}$.

Bacterial DNA extraction kits (OMEGA D3350-02) were used for DNA extraction from each isolate according to the manufacturer's instructions. Quantitative and qualitative analysis of genomic DNA was achieved using electrophoresis on $1 \%$ agarose gels and a TBS-380 mini fluorometer (Turner BioSystems Inc., Sunnyvale, CA). Only good quality DNA samples (OD260/280 $=1.8 \sim 2.0$, $>6 \mu \mathrm{g}$ ) were used to construct fragment libraries (200 to $300 \mathrm{bp})$.

Carbohydrates fermentation was tested using the API $50 \mathrm{CH}$ systems (Biomerieux, France) based on the manufacturer's instructions.

\section{Genome sequencing, assembly, prediction and annotation of coding sequences (CDS), and pathway mapping}

The ten genomes of the the Lactococcus type species/subspecies were sequenced using an Illumina HiSeq 2000 (Illumina Inc. USA) by generating paired-end libraries. The average length was $300 \mathrm{bp}$, and $838 \mathrm{Mb}$ of high quality data was generated for each isolate consistent with a sequencing depth of 129 - to 388-fold. Assembly of the paired-end reads was performed using SOAPdenovo v2 [24]. The software Gap Closer was used to fill the local inner gaps and correct single base errors (http://sourceforge.net/projects/soapdenovo2/files/GapCloser/). Gene prediction and functional annotation of predicted genes were achieved using RAST $2.0[25,26]$. The individual genome assemblies were deposited in the $\mathrm{NCBI}$, the accession

Table 1 List of the isolates used in the evaluation of the genus Lactococcus

\begin{tabular}{|c|c|c|c|c|}
\hline Type strains & Species & Source & Isolation Year & References \\
\hline DSM 22330 & Lactococcus chungangensis & activated sludge foam & 2008 & [46] \\
\hline JCM 16395 & Lactococcus fujiensis & vegetable matter & 2011 & [47] \\
\hline ATCC 19257 & Lactococcus lactis subsp. cremoris & cream & 1919 & [4] \\
\hline DSM 20450 & Lactococcus lactis subsp. hordniae & leaf hopper & 1977 & [4] \\
\hline ATCC 19435 & Lactococcus lactis subsp. lactis & milk & 1873 & [4] \\
\hline DSM 21502 & Lactococcus lactis subsp. tructae & $\begin{array}{l}\text { intestinal mucus of brown trout } \\
\text { (Salmo trutta) and rainbow trout }\end{array}$ & 2011 & [35] \\
\hline DSM 6634 & Lactococcus piscium & salmonid fish & 1990 & [48] \\
\hline DSM 20686 & Lactococcus plantarum & frozen peas & 1984 & [4] \\
\hline ATCC 43920 & Lactococcus raffinolactis & milk & 1932 & [4] \\
\hline DSM 20684 & Lactococcus garvieae & bovine mastitis & 1984 & [4] \\
\hline
\end{tabular}


numbers are shown in Table 2. Carbohydrate metabolism related pathway information was identified from the pathways described in the Kyoto Encyclopedia of Genes and Genomes (KEGG, http://www.kegg.jp/) [27].

\section{Core genome, pan-genome and unique gene analysis} For predicting the possible dynamic changes in genome size, the sizes of the core genome (common genes, mutually conserved), pan-genome (gene repertoire) and unique genes (specific genes, only found in one genome) were calculated. Owing to large variation were happened in the homologous genes between species within a genus, the concept of 'gene family' was used to take the place of the generally used term 'gene'. According to previous research $[28,29]$, the core genome, pan-genome and unique genes [30] of Lactococcus were estimated. A pair of genes was placed in the same gene family, when their amino acid sequences identity was $>50 \%$, and when more than $50 \%$ of the amino acid sequences of the longer gene could be covered by the shorter one.

For construction of the pan-genome, all of the predicted genes were first grouped into possible gene families for each genome and then the gene families of ten genomes were accumulated. The core genome was built by counting the number of commonly shared gene families within ten genomes, while the unique gene family was defined as the gene families that could only be found in one genome.

\section{The average nucleotide identity (ANI) and phylogenetic analysis}

The pair-wise ANI values of ten Lactococcus species/ subspecies were calculated using the methods of Goris et al. [30]. To infer phylogenetic relationships among Lactococcus species, we constructed a maximum likelihood (ML) tree based on the concatenated amino acid sequences of 643 core genes. Streptococcus $(S$.) thermophilus LMG 18311 was used as an out-group because it is phylogenetically closely related to the genus Lactococcus [31]. Amino acid sequences of the core genes were aligned by MUSCLE v3.8.31 [32], and the software PHYML was used to construct the ML tree with the WAG model and 500 bootstrap iterations [33]. In addition, a Neighbour-Joining (NJ) tree was inferred based on 16S rRNA sequences downloaded from Genbank for all ten type species/subspecies of Lactococcus using Mega 6.0 [34] (http://www.megasoftware.net) with 1000 bootstrap iterations; again S. thermophilus LMG 18311 was used as an out-group.

\section{Results}

\section{General features}

The estimated genomes of the type isolates of ten Lactococcus species/subspecies were sequenced and assembled into 43-226 scaffolds. Across species, the total genome size varied from 1.99 (L. plantarum) to 2.46 megabases (Mb; L. lactis subsp. lactis), with a mean value of $2.27 \mathrm{Mb}$ (Table 2). In addition, the $\mathrm{G}+\mathrm{C}$ content ranged from 34.81 (L. lactis subsp. hordniae) to $39.67 \%$ (L. raffinolactis) with an average value of $37.02 \%$ (Table 2 ). The numbers of predicted coding genes varied from $1861(L$. plantarum) to 2684 (L. lactis subsp. hordniae).

\section{Pan-genome and core genome analysis}

The pan-genome, defined as the full complement of genes from the ten Lactococcus genomes evaluated, contained 8036 gene families and grew continuously as each genome was added; a mean of 662 gene families were added for each genome (Fig. 1a). Compare to the pangene families, the amount of core gene families decreased sharply along with the number of genomes increased, reaching a minimum value of 643 for all ten genomes (Fig. 1b). The proportion of core genes varied between 23.9 (L. plantarum) and 34.6\% (L. lactis subsp. hordniae) of the total predicted coding genes for the ten

Table 2 Genomic features of species/ subspecies in the genus Lactococcus

\begin{tabular}{|c|c|c|c|c|c|}
\hline Type strains & Scaffold number & $\begin{array}{l}\text { Genome size } \\
(\mathrm{Mb})\end{array}$ & GC\% & $\begin{array}{l}\text { Predicated } \\
\text { coding genes }\end{array}$ & $\begin{array}{l}\text { Genbank accession } \\
\text { number }\end{array}$ \\
\hline L. chungangensis DSM $22330^{a}$ & 86 & 2.20 & 38.67 & 2154 & JXJT00000000 \\
\hline L. garvieae DSM 20684 & 71 & 2.02 & 38.52 & 1995 & JXJV00000000 \\
\hline L. fujiensis JCM 16395 & 69 & 2.08 & 36.95 & 2057 & JXJU00000000 \\
\hline L. lactis subsp. cremoris ATCC 19257 & 226 & 2.32 & 35.55 & 2477 & JXJZ00000000 \\
\hline L. lactis subsp. hordniae DSM 20450 & 101 & 2.43 & 34.81 & 2684 & JXKA00000000 \\
\hline L. lactis subsp. lactis ATCC 19435 & 76 & 2.46 & 35.23 & 2500 & JXKB00000000 \\
\hline L. lactis subsp. tructae DSM 21502 & 61 & 2.61 & 35.49 & 2612 & JXKC00000000 \\
\hline L. piscium DSM 6634 & 74 & 2.41 & 38.55 & 2301 & JXJW00000000 \\
\hline L. plantarum DSM 20686 & 43 & 1.99 & 36.76 & 1861 & JXJX00000000 \\
\hline L. raffinolactis ATCC 43920 & 121 & 2.21 & 39.67 & 2143 & JXJY00000000 \\
\hline
\end{tabular}

${ }^{\mathrm{a}}$ L. Lactococcus 

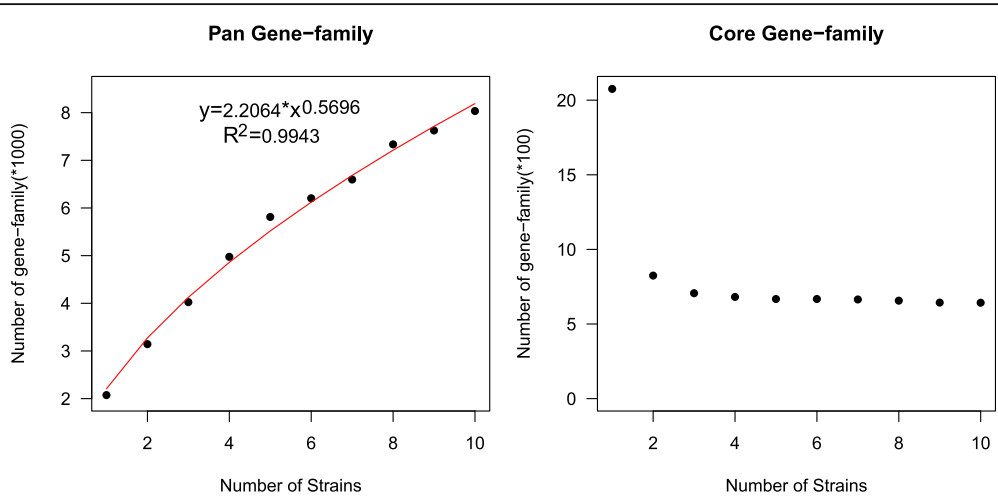

Fig. 1 Pan-genome and core genomes of species/subspecies in the Lactococcus genus. The gene accumulation curves show the number of new genes (pan-genome) and genes in common (core-genome) obtained by adding a new genome to a previous set. The pan-genome curve is the least squares fit of the power law to these values

Lactococcus species/subspecies. These observations indicated a high degree of genomic diversity among the ten type species/subspecies of the genus Lactococcus. Such genome diversity in the genus Lactococcus is not surprising considering the range of dairy, plant, and animal environments from which the species were isolated.

To examine the functional characteristics of the core genes, the Cluster of Orthologous Group (COG) database was used to investigate the distribution of core proteins mapped to each COG category (Fig. 2). There were eight subcategories in metabolism, seven subcategories in cellular processes and signaling, three subcategories in information storage and processing and two subcategories that were poorly characterized. The information storage and processing category accounted for $32.3 \%$ of the clusters, and the cellular processes and signaling category and metabolism categories accounted for 17.4 and $29.7 \%$ of clusters, respectively (Fig. 2). Furthermore, $18.66 \%$ of core genes were belong to translation, ribosomal structure, and biogenesis (J); $7.93 \%$ were involved in replication, recombination, and repair (L); and $6.22 \%$ were involved in cell wall/membrane/envelope biogenesis $(\mathrm{M})$. Other than the functional subcategories $\mathrm{J}, \mathrm{L}$, and $\mathrm{M}$, the 17 remaining functional subcategories included $6-38$ core genes. It is notable that most of the core genes played important roles in maintaining growth and reproduction in the genus

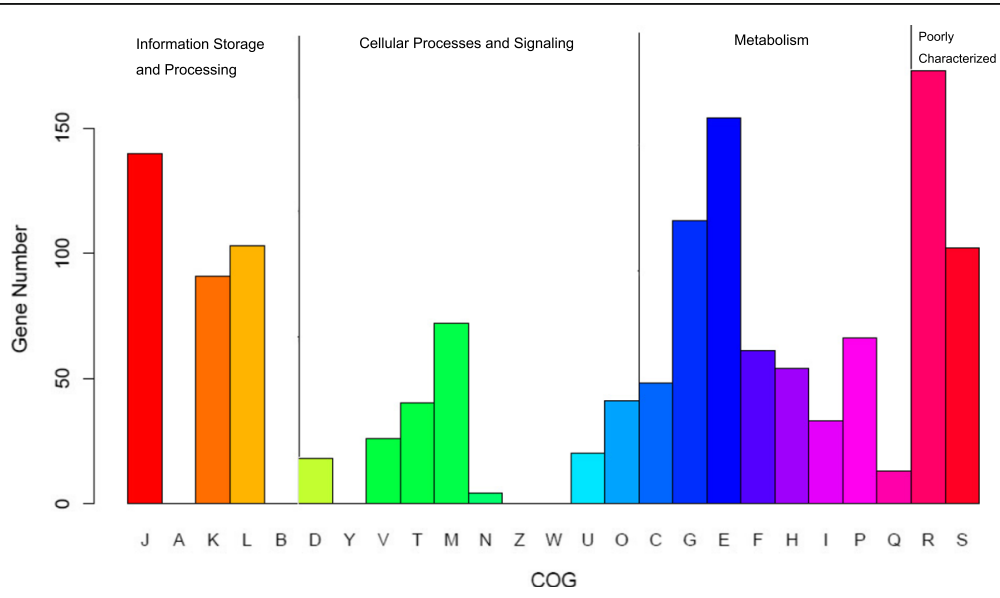

Fig. 2 Distribution of functional categories of core gene families in the Lactococcus genus. Cluster of Orthologous Group (COG) functional classification description — Information Storage and Processing: [A] RNA processing and modification; [B] chromatin structure and dynamics; [J] translation, ribosomal structure, and biogenesis; [K] transcription; [L] replication, recombination, and repair. Cellular Processes and Signaling: [D] cell cycle control, cell division, chromosome partitioning; [M] cell wall/membrane/envelope biogenesis; [N] cell motility; [O] posttranslational modification, protein turnover, chaperones; [T] signal transduction mechanisms; [U] intracellular trafficking, secretion, and vesicular transport; [V] defense mechanisms; [W] extracellular structures; [Y] nuclear structure; [Z] cytoskeleton. Metabolism: [C] energy production and conversion; [E] amino acid transport and metabolism; [F] nucleotide transport and metabolism; [H] coenzyme transport and metabolism; [G] carbohydrate transport and metabolism; [l] lipid transport and metabolism; [P] inorganic ion transport and metabolism; [Q] secondary metabolites biosynthesis, transport, and catabolism. Poorly Characterized: [R] general function prediction only; [S] function unknown 
Lactococcus; these genes are indispensable and constitute the basic framework of the Lactococcus genome.

\section{Phylogenetic relationships of Lactococcus strains}

In order to infer phylogenetic position of ten species within the genus Lactococcus, phylogenetic trees were constructed using the $16 \mathrm{~S}$ ribosomal RNA (rRNA) gene and the concatenated amino acid sequences of 643 core genes from the ten type isolates. The ML tree generated a reliable delineation of phylogenetic relationships across the Lactococcus species, because of more nodes were supported by $100 \%$ of the bootstrap iterations (Fig. 3a). According to this highly robust ML tree, the ten Lactococcus species/subspecies were divided into two branches; one branch contained L. raffinolactis ATCC $43920^{\mathrm{T}}$, L. chungangensis DSM $22330^{\mathrm{T}}$, L. plantarum DSM $20686^{\mathrm{T}}$, and L. piscium DSM $6634^{\mathrm{T}}$, while the other branch included four L. lactis subspecies, L. fujiensis JCM $16395^{\mathrm{T}}$, and L. garvieae DSM20684 ${ }^{\mathrm{T}}$.

The topologies of the 16S rRNA gene trees were similar to the ML tree (Fig. 3b). However, the resolution and accuracy of the tree of concatenated amino acid sequences was better than that of the 16S rRNA gene tree. For instance, ATCC $19257^{\mathrm{T}}$ and L. lactis subsp. tructae DSM $21502^{\mathrm{T}}$, L. lactis subsp. hordniae DSM $20450^{\mathrm{T}}$ and L. lactis subsp. lactis ATCC $19435^{\mathrm{T}}$ were clustered closely in the 16S rRNA gene tree and shared $>99.90 \%$ identity, but were divided in the orthologous proteins tree. Both trees proved that the ten isolates clustered together to form a single genus.

\section{Genetic diversity and species taxonomy}

The ANI value is always used to estimate the genetic distance between isolates, which means the sequence identities of the conserved regions between two genomes [29]. The pair-wise ANI values of ten Lactococcus species/ subspecies were calculated to study the interspecies genetic relatedness within the genus Lactococcus. Two clusters formed when the ANI for Lactococcus species was analyzed (Fig. 4). One cluster contained two subgroups: subgroup I (L. lactis subsp. hordniae and $L$. lactis subsp. lactis) and subgroup II (L. lactis subsp. cremoris and L. lactis subsp. tructae). The 16S rRNA gene identity for the four subspecies was $>99 \%$ [35]; however, the ANI value for this cluster was $86.1-97.9 \%$. It is worth noting that the pairwise ANI values between the two subgroups range from $86.1-86.5 \%$, below the recommended 95\% threshold value for species circumscription [36]. To further determine the relationships between the four subspecies, we performed additional comparative genomics with 15 genomic sequences of $L$. lactis subsp. lactis and L. lactis subsp. cremoris from NCBI. The ANI value distribution of Lactococcus is shown in Additional file 1: Table S1, and the heat map

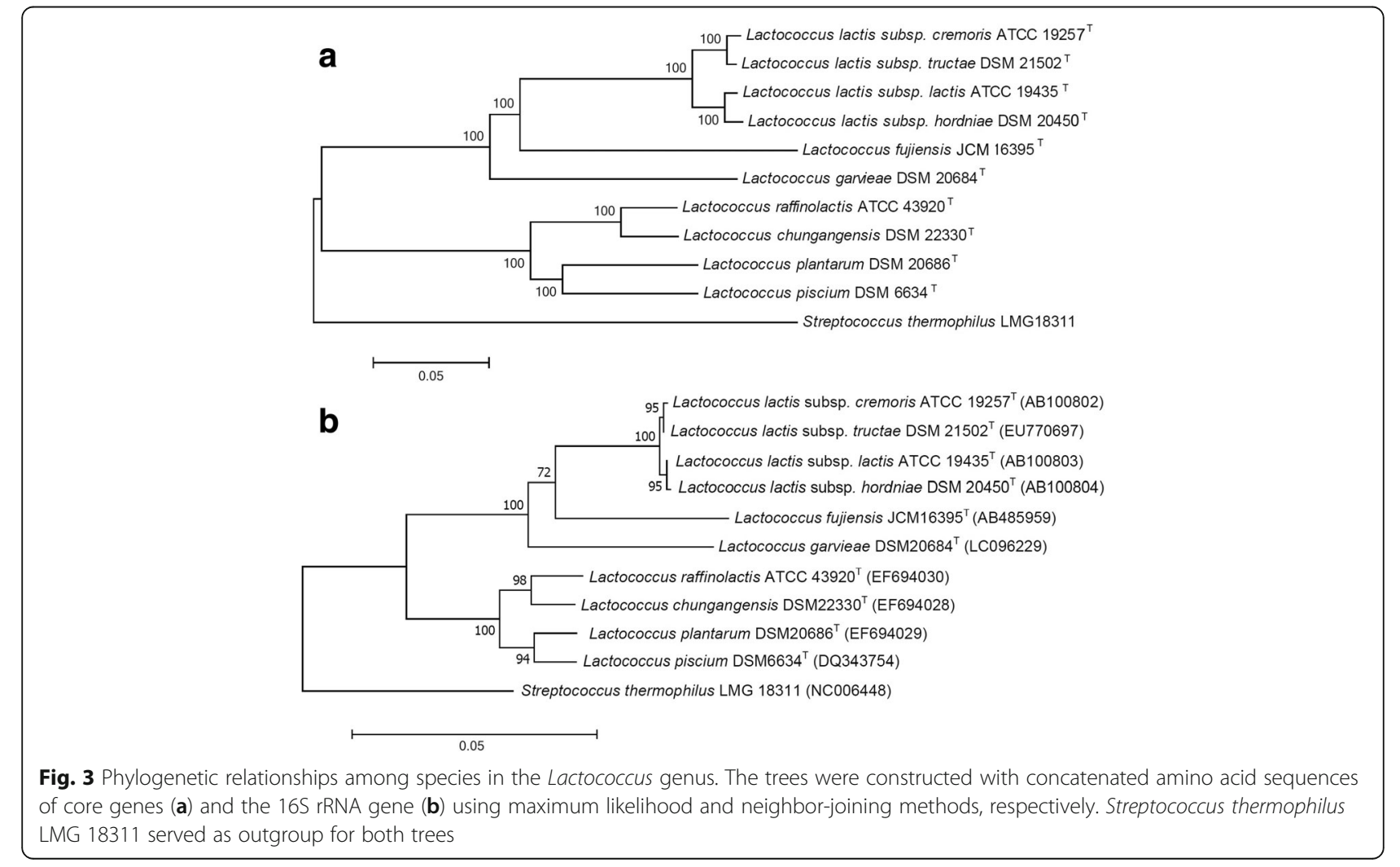




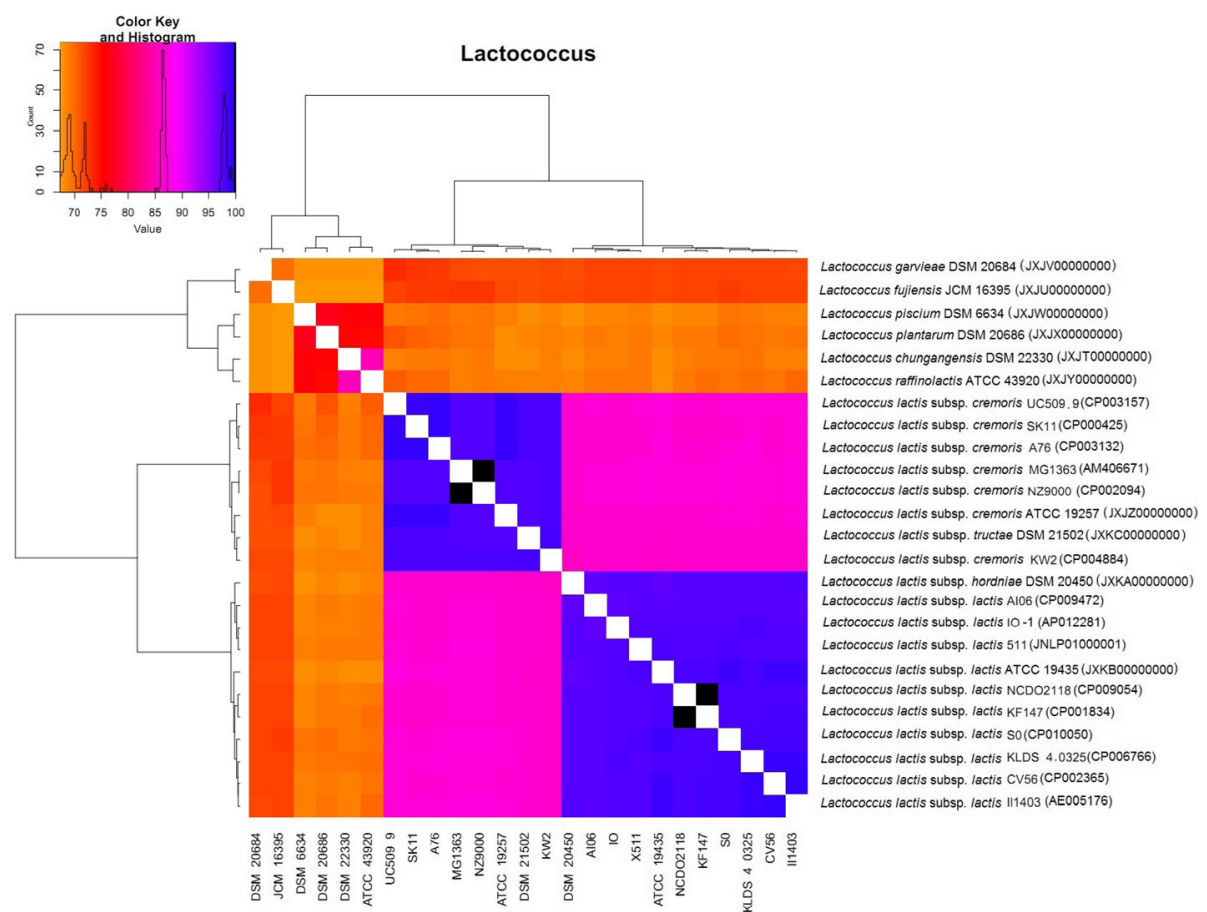

Fig. 4 Pairwise average nucleotide identity (ANI) values across ten Lactococcus genomes. The colours in the heat map represent pairwise ANI values, with a gradient from yellow (low identity) to blue (high identity)

was built based on pairwise ANI values (Fig. 4). Based on these results, the four strains were clearly divided into two subgroups, and these values were in line with the DNADNA hybridization results. DNA relatedness values between the type strains L. lactis subsp. tructae and L. lactis subsp. hordniae, L. lactis subsp. lactis, L. lactis subsp. cremoriswere 60,62 , and $90 \%$, respectively [35].

Therefore, we suggest that L. lactis subsp. cremoris ATCC $19257^{\mathrm{T}}$ and L. lactis subsp. tructae DSM $21502^{\mathrm{T}}$ should be separated from L. lactis subsp. hordniae DSM $20450^{\mathrm{T}}$ and L. lactis subsp. lactis ATCC $19435^{\mathrm{T}}$ as a single species on the basis of the genetic distance and the phylogenetic relationships shown in ML tree (Fig. 3a). The other cluster contained six type isolates with $16 \mathrm{~S}$ rRNA gene identity of $91-97 \%$ and ANI of $67.4-85.0 \%$. These are the classical delimitation criteria for different species within one genus.

\section{Carbohydrate metabolism}

Carbohydrate metabolism plays a key role in the growth of Lactococcus species. On average, $12.5 \%$ of the genes in each Lactococcus genome are associated with carbohydrate metabolism. Among the core genes of the ten Lactococcus species/subspecies evaluated, a total of 38 genes (5.9\%) were associated with carbohydrate metabolism. The majority were involved in glycolysis/gluconeogenesis and the pentose phosphate pathway (PPP). Twenty-eight key enzymes that contribute to glycolysis/gluconeogenesis and PPP were encoded (Table 3, Additional file 2: Figure S1). Our results indicated that glycolysis/gluconeogenesis and the PPP are the central carbohydrate metabolism pathways of Lactococcus species. In addition, all strains have genes encoding fructokinase (EC 2.7.1.4), phosphoenolpyruvate-protein phosphotransferase (EC 2.7.1.199), and glucose-6-phosphate isomerase (EC 5.3.1.9), which are key enzymes in fructose and mannose metabolism. Thus, all strains could ferment D-glucose, D-fructose, and D-mannose (Table 4). Pathways enabling the use of L-arabinose, L-sorbose, L-rhamnose, xylitol, D-lyxose, and D-tagatose as energy were not found in the genome sequences of the ten strains examined.

Some differences in carbohydrate metabolism genes were also found among the ten Lactococcus species/subspecies. The number of carbohydrate metabolism genes in the ten species/subspecies evaluated varied from 194 (L. plantarum DSM 20686 ${ }^{\mathrm{T}}$ ) to 366 (L. lactis subsp. tructae DSM $21502^{\mathrm{T}}$ ). Pairwise comparisons among the ten Lactococcus species/subspecies showed that the number of strain-specific carbohydrate metabolism genes varied between 2 and 15, and appeared to reflect the functional diversity observed for each isolate. To detect differences in functional genes related to carbohydrate metabolism, acid production from carbohydrates 
Table 3 Critical enzymes were coded by core genes of ten Lactococcus species/ subspecies for contributing to the glycolysis/ gluconeogenesis and pentose phosphate pathway

\begin{tabular}{|c|c|c|}
\hline No. & EC Number & enzymes \\
\hline 1 & EC 1.1.1.1 & Alcohol dehydrogenase \\
\hline 2 & EC 1.1.1.27 & L-lactate dehydrogenase \\
\hline 3 & EC 1.1.1.44 & $\begin{array}{l}\text { 6-phosphogluconate dehydrogenase, } \\
\text { decarboxylating }\end{array}$ \\
\hline 4 & EC 1.1.1.49 & Glucose-6-phosphate 1-dehydrogenase \\
\hline 5 & EC 1.2.1.12 & glyceraldehyde-3-phosphate dehydrogenase \\
\hline 6 & EC 1.2.4.1 & pyruvate dehydrogenase E1 component \\
\hline 7 & EC 1.2.7.1 & Pyruvate-flavodoxin oxidoreductase \\
\hline 8 & EC 1.8.1.4 & dihydrolipoamide dehydrogenase \\
\hline 9 & EC 2.2.1.1 & Transketolase \\
\hline 10 & EC 2.3.1.12 & Dihydrolipoamide acetyltransferase \\
\hline 11 & EC 2.7.1.11 & 6-phosphofructokinase \\
\hline 12 & EC 2.7.1.2 & Glucokinase \\
\hline 13 & EC 2.7.1.40 & pyruvate kinase \\
\hline 14 & EC 2.7.1.40 & Pyruvate kinase \\
\hline 15 & EC 2.7.2.3 & Phosphoglycerate kinase \\
\hline 16 & EC 2.7.6.1 & Ribose-phosphate pyrophosphokinase \\
\hline 17 & EC 3.1.1.31 & 6-phosphogluconolactonase \\
\hline 18 & EC 3.1.3.11 & Fructose-1,6-bisphosphatase \\
\hline 19 & EC 3.2.1.86 & 6-phospho-beta-glucosidase \\
\hline 20 & EC 4.1.2.13 & Fructose-bisphosphate aldolase class II \\
\hline 21 & EC 4.2.1.11 & Enolase \\
\hline 22 & EC 5.1.3.1 & Ribulose-phosphate 3-epimerase \\
\hline 23 & EC 5.1.3.3 & Aldose 1-epimerase \\
\hline 24 & EC 5.3.1.1 & Triosephosphate isomerase \\
\hline 25 & EC 5.3.1.6 & Ribose 5-phosphate isomerase A \\
\hline 26 & EC 5.3.1.9 & Glucose-6-phosphate isomerase \\
\hline 27 & EC 5.4.2.11 & $\begin{array}{l}\text { 2,3-bisphosphoglycerate-dependent } \\
\text { phosphoglycerate mutase }\end{array}$ \\
\hline 28 & EC 5.4.2.7 & Phosphopentomutase \\
\hline
\end{tabular}

was tested using the API $50 \mathrm{CH}$ system (bioMérieux, Inc., Marcy l'Etoile, France) (Table 4). Raffinose can be enzymatically hydrolyzed to melibiose by $\beta$ fructofuranoside (EC 3.2.1.26), and melibiose is further hydrolyzed to D-glucose ( $\alpha$-galactosidase; EC 3.2.1.22), and enters the glycolysis pathway. Three strains, L. lactis subsp. tructae DSM $21502^{\mathrm{T}}$, L. piscium DSM $6634^{\mathrm{T}}$, and L. raffinolactis ATCC $43920^{\mathrm{T}}$, can utilize raffinose and melibiose, because they possess the key enzymes (EC 3.2.1.26 and EC 3.2.1.22) for raffinose and melibiose metabolism, while L. plantarum DSM $20686^{\mathrm{T}}$, L. lactis subsp. hordniae DSM $20450^{\mathrm{T}}$, and L. chungangensis DSM $22330^{\mathrm{T}}$ with $\beta$-fructofuranoside and no $\alpha$ galactosidase, cannot utilize raffinose and melibiose.
Furthermore, $\beta$-fructofuranoside is a key enzyme for sucrose hydrolysis, and so the four strains lacking this enzyme cannot utilize sucrose. The other six strains can hydrolyze sucrose to D-glucose-6P and D-fructose. The role of $L$. lactis as an important industrial starter strain is mainly due to the rapid conversion of lactose to lactic acid. Lactose is hydrolyzed by $\beta$-galactosidase (EC 3.2.1.23) to give $\alpha$-D-glucose and D-galactose, and it can also be catalyzed to lactose 6-phosphate (Lac-6P) by lactose phosphotransferase (EC 2.7.1.207). Lac-6P is subsequently hydrolyzed by 6 -phospho- $\beta$-galactosidase (EC 3.2.1.85) to give galactose 6-phosphate (Gal-6P), and Gal-6P is catabolized by the tagatose 6-phosphate (Tag$6 \mathrm{P})$ pathway $[37,38]$. Comparative genomic analysis showed that most strains have the key enzymes for lactose metabolism, with the exception of L. chungangensis DSM $22330^{\mathrm{T}}$, L. fujiensis $\mathrm{JCM} 16395^{\mathrm{T}}$, L. plantarum DSM $20686^{\mathrm{T}}$, and L. lactis subsp. tructae DSM $21502^{\mathrm{T}}$. These four strains lack lactose phosphotransferase and $\beta$-galactosidase, but L. lactis subsp. tructae DSM $21502^{\mathrm{T}}$ and $L$. fujiensis JCM $16395^{\mathrm{T}}$ could utilize lactose in the API 50 test (Table 4). Therefore, we inferred that they have additional pathways for lactose metabolism. Ribokinase (EC 2.7.1.15) is the key enzyme in D-ribose metabolism. L. raffinolactis ATCC $43920^{\mathrm{T}}$, L. plantarum DSM $20686^{\mathrm{T}}$, L. lactis subsp. hordniae DSM $20450^{\mathrm{T}}$, and L. chungangensis DSM $22330^{\mathrm{T}}$ have no genes encoding ribokinase, so they cannot utilize D-ribose.

\section{Discussion}

It is considerable realistic significance for applying Lactococcus strains to systematically analyze the evolutionary history and phylogenetic position of the Lactococcus species/subspecies. Therefore, we completed the wholegenome sequencing of ten type strains in Lactococcus genus to assess their genomic characteristics, metabolic diversity, and phylogenetic relationships.

Across ten Lactococcus species, the total genome size varied from 1.99 (L. plantarum) to $2.46 \mathrm{Mb}$ (L. lactis subsp. lactis) (Table 2). The genome size of the Lactococcus species evaluated was relatively small compared with other LAB genera, such as Enterococcus (2.31-5.27 Mb) and Lactobacillus (1.23-4.91 Mb) [32]. A smaller genome size was suggested to indicate adaptations for reproductive efficiency or competitiveness in new environments [39]. The $\mathrm{G}+\mathrm{C}$ content of 10 strains ranged from 34.81 (L. lactis subsp. hordniae) to $39.67 \%$ (L. raffinolactis). The $\mathrm{G}+\mathrm{C}$ content of genome is influenced by selection and mutation involving multiple factors, including the symbiotic lifestyle, environment, nitrogen fixation ability, aerobiosis, and how the polIIIa subunits are combined [40]. In addition, analysis of the trends in pan-genome size of Lactococcus species/subspecies proved that Lactococcus genus has an open pan-genome. 
Table 4 Phenotypic characteristics of ten type strains in Lactococcus genus

\begin{tabular}{|c|c|c|c|c|c|c|c|c|c|c|}
\hline Carbohydrate & DSM 22330 & DSM 20684 & JCM 16395 & ATCC 19257 & DSM 20450 & ATCC 19435 & DSM 21502 & DSM 6634 & DSM 20686 & ATCC 43920 \\
\hline control & - & - & - & - & - & - & - & - & - & - \\
\hline L-Arabinose & - & - & - & - & - & - & - & - & - & - \\
\hline Ribose & - & + & + & - & - & + & + & - & - & - \\
\hline D-xylose & - & - & - & - & - & + & - & + & - & + \\
\hline D-Glucose & + & + & + & + & + & + & + & + & + & + \\
\hline D-Fructose & + & + & + & + & + & + & + & + & + & + \\
\hline D-Mannose & + & + & + & + & + & + & $w^{a}$ & + & + & + \\
\hline L-Sorbose & - & - & - & - & - & - & - & - & - & - \\
\hline L-Rhamnose & - & - & - & - & - & - & - & - & - & - \\
\hline D-Sorbitol & - & - & - & - & - & - & - & - & + & - \\
\hline Amygdalin & + & + & - & - & - & w & + & + & + & - \\
\hline D-Cellobiose & + & + & + & - & + & + & + & + & + & + \\
\hline D-Maltose & + & + & + & - & + & + & + & + & + & + \\
\hline D-Lactose & - & + & + & + & + & + & + & + & - & + \\
\hline D-Melibiose & - & - & - & - & - & - & + & + & - & + \\
\hline D-Sucrose & + & - & - & - & + & - & + & + & + & + \\
\hline D-Trehalose & + & + & + & - & - & + & + & + & + & + \\
\hline D-Raffinose & - & - & - & - & - & - & + & + & - & + \\
\hline Xylitol & - & - & - & - & - & - & - & - & - & - \\
\hline D-Lyxose & - & - & - & - & - & - & - & - & - & - \\
\hline D-Tagatose & - & - & - & - & - & - & - & - & - & - \\
\hline
\end{tabular}

${ }^{a} w$ weakly positive

This may be associated with the range of environments colonized by different Lactococcus species and the existence of numerous ways of exchanging genetic material. Previous reports showed that bacterial genomes change when they adapt to variable conditions, and that greater niche diversity requires larger pan-genomes [41].

Currently, the $16 \mathrm{~S}$ rRNA gene sequencing technique and genomic DNA-DNA hybridization are considered the gold standards for identification of isolates at the species or genus level. However, because of conservative property, $16 \mathrm{~S}$ rRNA gene could provide sufficient resolution at the species levels [42, 43], and DNA-DNA hybridization is rather time-consuming and labor-intensive. With advances in sequencing and computational technologies, the combination of core genome phylogeny and ANI values could provide accurate taxonomic guidance for LAB based on whole-genome sequences [44]. The lowest value for the Lactococcus genus was $67.4 \%$, while the highest ANI value was $97.9 \%$, revealing that the genomic sequence identities between different pairs of species had marked differences. The ANI values were mainly distributed in the region of $67-$ 68\% (Additional file 1: Table S1), while the distribution of
Bifidobacterium genus was concentrated in the region of $71-74 \%$ [29]. These results suggest that the majority of sequence identities between Lactococcus genomes were low, only slightly higher than those between species from different genera. This could be used as a reference characteristic for identification of Lactococcus species.

Differences in carbohydrate metabolism genes among the ten Lactococcus species/subspecies showed that each could utilize various different carbohydrates. One interesting application of comparative genomics is the identification of associations between strains and their origins. Previous studies using comparative genomics in the genera Nocardiopsis [45] and Bifidobacterium [29] demonstrated specificity to particular ecological niches. In this study, the genotypic and phenotypic carbohydrate metabolism analyses suggested that the lactococcal species may correlate with respect to their origins, but regularities were not found. This is probably due to the unequal and small numbers of isolates from different origins. Three species were isolated from milk and four species from fish, bovine, and leafhopper, whereas only two species were isolated from plants and one species from activated sludge foam (Table 1). As more species are 
discovered from different countries and origins, further genomic evidence may become available for the genus Lactococcus, which would provide strong indications of the factors that have affected its evolutionary history. The majority of the carbohydrate fermentation results are in accordance with the metabolism pathway predicted by the genome sequence, but some differences were also detected, for which there are at least two explanations: predicted gene function based on sequence data is not completely accurate, and the pathways of carbohydrate metabolism are not yet well understood in KEGG.

\section{Conclusion}

In the present study, ten type isolates of species/subspecies from the genus Lactococcus were sequenced and analyzed. Comparative genomic analysis revealed that species in the genus Lactococcus had relatively small and diverse genomes. The high degrees of diversity in genome size, gene content, and carbohydrate metabolism may be because the isolates originated from a wide range of host types and ecological niches. The genus Lactococcus has an open pan-genome, and thus the size of the pan-genome is as yet underestimated and will increase as additional isolates and species are sequenced. Since we had only a limited number of isolates, gene evolution in the genus Lactococcus was not analyzed. Nonetheless, this study provided insights into genomic and metabolic diversity and phylogenetic relationships of the majority of type species/subspecies in the genus Lactococcus, which will lay the foundation for future studies of their taxonomy and functional genomics.

\section{Additional files}

Additional file 1: Table S1. Glycolysis/gluconeogenesis and the pentose phosphate pathway of ten type strains in the Lactococcus genus. The names of the genes that were present in the genomes of ten type strains in Lactococcus genus are shown in red, and those that were absent in all strains are shown in gray. (JPEG $336 \mathrm{~kb}$ )

Additional file 2: Figure S1. Pairwise ANI values across ten Lactococcus genomes. (DOC $42 \mathrm{~kb}$ )

\section{Abbreviations \\ ANI: Average nucleotide identity; CDS: Coding sequences; KEGG: Kyoto Encyclopedia of Genes and Genomes; L.: Lactococcus; LAB: Lactic acid bacterium; NCBI: National Center for Biotechnology Information; S.: Streptococcus}

\section{Acknowledgements}

The authors are indebted to the members of the Key Laboratory of Dairy Biotechnology and Engineering, Inner Mongolia Agricultural University, Hohhot, for their technical supports.

\section{Funding}

This research was supported by the National Natural Science Foundation of China (Beijing, China; Grant No. 31622043) and National Natural Science Foundation of China (Beijing, China; Grant No.31601454).

\section{Availability of data and materials}

The sequence information of ten Lactococcus type species in this paper has been deposited in the National Center for Biotechnology Information (NCBI; http://www.ncbi.nlm.nih.gov), the accession numbers JXKA00000000,

JXKB00000000, JXKC00000000, JXJW00000000, JXJX00000000, JXJT00000000 JXJU00000000, JXJV00000000, JXJZ00000000 and JXJY00000000.

\section{Authors' contributions}

$J Y$ and ZHS designed the experiments. YTQ and YR performed the experiments. YQS and WJL analyzed the data. JY drafted the manuscript. All authors read and approved the final manuscript.

\section{Ethics approval and consent to participate}

The Ethical Committee of the Inner Mongolia Agricultural University ruled that no formal ethics approval was required for this study.

\section{Consent for publication}

Not applicable.

\section{Competing interests}

The authors declare that they have no competing interests.

\section{Publisher's Note}

Springer Nature remains neutral with regard to jurisdictional claims in published maps and institutional affiliations.

Received: 13 July 2017 Accepted: 19 October 2017

Published online: 03 November 2017

\section{References}

1. Sun Z, Yu J, Dan T, Zhang W, Zhang H. Phylogenesis and evolution of lactic acid bacteria. In: Zhang H, Cai Y, editors. Lactic acid bacteria. Netherlands: Springer; 2014. p. 1-101

2. Sakala RM, Hayashidani $H$, Kato $Y$, Hirata $T$, Makino $Y$, Fukushima A, Yamada T, Kaneuchi C, Ogawa M. Change in the composition of the microflora on vacuum-packaged beef during chiller storage. Int J Food Microbiol. 2002; 74(1-2):87-99.

3. Teuber M. Genus II. Lactococcus Schleifer, Kraus, Dvorak, Kilpper-Balz,Collins and Fischer 1986. In: De Vos P, Garrity GM, Jones D, Krieg NR, Ludwig W Rainey FA, Schleifer KH, Whitman WB, editors. Bergey's manual of systematic bacteriology, vol. 3. Berlin: Springer; 2009. p. 711-22.

4. Schleifer KH, Kraus J, Dvorak C, Kilpper-Bälz R, Collins MD, Fischer W. Transfer of Streptococcus lactis and related streptococci to the genus Lactococcus gen. Nov. Syst Appl Microbiol. 1985;6(2):183-95.

5. Park SH, Itoh K, Kikuchi E, Niwa H, Fujisawa T. Identification and characteristics of nisin Z-producing Lactococcus lactis subsp. lactis isolated from Kimchi. Curr Microbiol. 2003:46(5):385-8.

6. Rattanachaikunsopon P, Phumkhachorn P. Lactic acid bacteria: their antimicrobial compounds and their uses in food production. Ann Biol Res. 2010;1(4):218-28.

7. Morello E, Bermúdez-Humarán LG, Llull D, Solé V, Miraglio N, Langella P, Poquet I. Lactococcus lactis, an efficient cell factory for recombinant protein production and secretion. J Mol Microbiol Biotechnol. 2008;14(1-3):48-58.

8. Fang $H$, Elina $T$, Heikki A, Seppo S. Modulation of humoral immune response through probiotic intake. FEMs Immunol Med Mic. 2000;29:47-52.

9. Yadav K, Bhardwaj A, Kaur G, Iyer R, De S, Kumar N, Malik RK. Potential of Lactococcus lactis as a probiotic and functional lactic acid bacteria in dairy industry. Int J Probiotics Prebiotics. 2009:4:219-28.

10. Teixeira LM, Merquior VL, Vianni MC, Carvalho MG, Fracalanzza SE, Steigerwalt AG, Brenner DJ, Facklam RR. Phenotypic and genotypic characterization of atypical Lactococcus garvieae strains isolated from water buffalos with subclinical mastitis and confirmation of L. garvieae as a senior subjective synonym of Enterococcus seriolicida. Int J Syst Bacteriol. 1996;46(3):664-8.

11. Alegría A, Alvarezmartín P, Sacristán N, Fernández E, Delgado S, Mayo B. Diversity and evolution of the microbial populations during manufacture and ripening of Casín, a traditional Spanish, starter-free cheese made from cow's milk. Int J Food Microbiol. 2009;136:44-51.

12. Fortina MG, Ricci G, Acquati A, Zeppa G, Gandini A, Manachini PL. Genetic characterization of some lactic acid bacteria occurring in an artisanal 
protected denomination origin (PDO) Italian cheese, the Toma piemontese. Food Microbiol. 2003;20(02):397-404.

13. Foschino R, Picozzi C, Borghi M, Cerliani MC, Cresci E. Investigation on the microflora of Caprino Lomeardo cheese from raw goat milk.ltal. J Food Sci. 2006;18(1):33-49.

14. Yu J, Wang HM, Zha MS, Qing YT, Bai N, Ren Y, Xi XX, Liu WJ, Menghe BLG, Zhang HP. Molecular identification and quantification of lactic acid bacteria in traditional fermented dairy foods of Russia. J Dairy Sci. 2015; 98(8):5143-454

15. Touchon M, Cury J, Yoon EJ, Krizova L, Cerqueira GC, Murphy C, Feldgarden M, Wortman J, Clermont D, Lambert T. The genomic diversification of the whole Acinetobacter genus: origins, mechanisms, and consequences. Genome Biol Evol. 2014;6(10):2866-82.

16. Gao XY, Zhi XY, Li HW, Klenk HP, Li WJ. Comparative genomics of the bacterial genus Streptococcus illuminates evolutionary implications of species groups. PLoS One. 2014;9:e101229.

17. Kant R, Blom J, Palva A, Siezen RJ, de Vos WM. Comparative genomics of Lactobacillus. J Microbial Biotechnol. 2010;4(3):323-32.

18. Lugli GA, Milani C, Turroni F, Duranti S, Ferrario C, Viappiani A, Mancabelli L, Mangifesta M, Taminiau B, Delcenserie V, van Sinderen D, Ventura M. Investigation of the evolutionary development of the genus Bifidobacterium by comparative genomics. Appl Environ Microbiol. 2014;80:6383-94.

19. Bolotin A, Wincker P, Mauger S, Jaillon O, Malarme K, Weissenbach J, Ehrlich $\mathrm{SD}$, Sorokin A. The complete genome sequence of the lactic acid bacterium Lactococcus lactis ssp. lactis IL1403. Genome Res. 2001;11(5):731-53.

20. Yang CH, Wu CC, Cheng WS, Chung MC, Tsai YC, Chang CH. A17, the first sequenced strain of Lactococcus lactis subsp. cremoris with potential immunomodulatory functions. Genome Announc. 2015;3(1):e01563-14.

21. Andreevskaya M, Johansson $P$, Laine $P$, Smolander OP, Sonck M, Rahkila R, Jääskeläinen E, Paulin L, Auvinen P, Björkroth J. Genome sequence and transcriptome analysis of meat spoilage lactic acid bacterium Lactococcus piscium MKFS47. Appl Environ Microbiol. 2015:81:3800-11.

22. Zhao F, Ma H, Ying L, Teng K, Kang X, Wang F, Yang X, Jin Z. Complete genome sequence of Lactococcus lactis SO, an efficient producer of nisin. J Biotechnol. 2015;198:15-6.

23. Meslier V, Loux V, Renault P. Genome sequence of Lactococcus raffinolactis strain 4877 , isolated from natural dairy starter culture. J Bacteriol. 2012:194(22):6364

24. Luo R, Liu B, Xie Y, Li Z, Huang W, Yuan J, He G, Chen Y, Qi P, Liu Y. SOAPdenovo2: an empirically improved memory-efficient short-read de novo assembler. Gigascience. 2012;1(1):1-6.

25. Aziz RK, Bartels D, Best AA, DeJongh M, Disz T, Edwards RA, Formsma K, Gerdes S, Glass EM, Kubal M. The RAST server: rapid annotations using subsystems technology. BMC Genomics. 2008;9:75.

26. Overbeek R, Olson R, Pusch GD, Olsen GJ, Davis JJ, Disz T, Edwards RA Gerdes S, Parrello B, Shukla M, et al. The SEED and the rapid annotation of microbial genomes using subsystems technology (RAST). Nucleic Acids Res. 2014;42(Database issue):D206-14

27. Kanehisa M, Sato Y, Kawashima M, Furumichi M, Mao T. KEGG as a reference resource for gene and protein annotation. Nucleic Acids Res. 2015;44(D1):D457-62.

28. Lukjancenko O, Ussery DW, Wassenaar TM. Comparative genomics of Bifidobacterium, Lactobacillus and related probiotic genera. Microb Ecol. 2012;63(3):651-73.

29. Sun Z, Zhang W, Guo C, Yang $X$, Liu WJ, Wu YR, Song YQ, Kwok LY, Cui YJ, Menghe BLG, Yang TF, Hu LP, Zhang HP. Comparative genomic analysis of 45 type strains of the genus Bifidobacterium: a snapshot of its genetic diversity and evolution. PLoS One. 2015;10(2):e0117912.

30. Goris J, Konstantinidis KT, Klappenbach JA, Coenye T, Vandamme P, Tiedje JM. DNA-DNA hybridization values and their relationship to whole-genome sequence similarities. Int J Syst Evol Microbiol. 2007;57(1):81-91.

31. Sun Z, Harris HMB, Mccann A, Guo C, Argimón S, Zhang W, Yang X, Jeffery IB, Cooney JC, Kagawa TF. Expanding the biotechnology potential of lactobacilli through comparative genomics of 213 strains and associated genera. Nat Commun. 2015;6:8322.

32. Edgar RC. MUSCLE: a multiple sequence alignment method with reduced time and space complexity. BMC bioinformatics. 2004;5:113.

33. Guindon S, Dufayard JF, Lefort V, Anisimova M, Hordijk W, Gascuel O. New algorithms and methods to estimate maximum-likelihood phylogenies: assessing the performance of PhyML 3.0. Syst Biol. 2010;59(3):307-21.
34. Kumar S, Stecher G, Tamura K. MEGA7: molecular evolutionary genetics analysis version 7.0 for bigger datasets. Mol Biol Evol. 2016;33(7):1870.

35. Pérez T, Balcázar JL, Peix A, Valverde A, Velázquez E, De BI, Ruizzarzuela I. Lactococcus lactis subsp. tructae subsp. nov. isolated from the intestinal mucus of brown trout (Salmo Trutta) and rainbow trout (Oncorhynchus Mykiss). Int J Syst Evol Microbiol. 2011;61(8):1894-8.

36. Richter M, Rosselló-Móra R. Shifting the genomic gold standard for the prokaryotic species definition. Proc Natl Acad Sci U S A. 2009;106(45):19126-31.

37. Benthin S, Nielsen J, Villadsen J. Galactose expulsion during lactose metabolism in Lactococcus lactis subsp. cremoris FD1 due to dephosphorylation of intracellular galactose 6-phosphate. Appl Environ Microbiol. 1994;60(4):1254-9.

38. Lorca G, Reddy L, Nguyen A, Sun El, Tseng J, Yen MR, Saier MH, Milton H, Saier J. Lactic acid bacteria: comparative genomic analyses of transport systems. In: Mozzi F, Raya RR, Vignolo GM (eds) Biotechnology of lactic acid bacteria: novel applications. Oxford: Wiley-Blackwell. 2010. pp. 73-87. doi:10.1002/9780813820866.ch1.

39. Burke GR, Moran NA. Massive genomic decay in Serratia symbiotica, a recently evolved symbiont of aphids. Genome Biol Evol. 2011;3(5):195-208

40. Hao W, Zhang Z, Hu S, Yu J. On the molecular mechanism of GC content variation among eubacterial genomes. Biol Direct. 2012;7(1):2.

41. Konstantinidis KT, Tiedje JM. Trends between gene content and genome size in prokaryotic species with larger genomes. Proc Natl Acad Sci U S A. 2004;101(9):3160-5.

42. Weisburg WG, Barns SM, Pelletier DA, Lane DJ. 16 S ribosomal DNA amplification for phylogenetic study. J Bacteriol. 1991:173(2):697-703.

43. Stackebrandt E, Goebel BM. Taxonomic note: a place for DNA-DNA reassociation and 165 rRNA sequence analysis in the present species definition in bacteriology. Int J Syst Bacteriol. 1994;44(4):846-9.

44. Xu J. Microbial ecology in the age of genomics and metagenomics: concepts, tools, and recent advances. Mol Ecol. 2006;15(7):9-10.

45. Li HW, Zhi XY, Yao JC, Zhou Y, Tang SK, Klenk HP, Zhao J, Li WJ. Comparative genomic analysis of the genus Nocardiopsis provides new insights into its genetic mechanisms of environmental adaptability. PLoS One. 2013:8(4):e61528.

46. Cho SL, Nam SW, Yoon JH, Lee JS, Sukhoom A, Kim W. Lactococcus chungangensis sp. nov., a lactic acid bacterium isolated from activated sludge foam. Int J Syst Evol Microbiol. 2008;58(Pt 8):1844-9.

47. Cai Y, Yang J, Pang H, Kitahara M. Lactococcus fujiensis sp. nov., a lactic acid bacterium isolated from vegetable matter. Int J Syst Evol Microbiol. 2011; 61(Pt7):1590-4.

48. Williams AM, Fryer $J$, Collins MD. Lactococcus piscium sp. nov. a new Lactococcus species from salmonid fish. FEMS Microbiol Letters. 1990; 68(1-2):109-13.

\section{Submit your next manuscript to BioMed Central and we will help you at every step:}

- We accept pre-submission inquiries

- Our selector tool helps you to find the most relevant journal

- We provide round the clock customer support

- Convenient online submission

- Thorough peer review

- Inclusion in PubMed and all major indexing services

- Maximum visibility for your research

Submit your manuscript at www.biomedcentral.com/submit 